\title{
TREATMENT OF CORNEAL INFECTIONS DUE TO PSEUDOMONAS PYOCYANEUS WITH POLYMYXIN B SULPHATE OINTMENT*
}

\author{
BY \\ D. P. GURD \\ Medical Department of the Navy
}

THE infection of the cornea with Pseudomonas pyocyaneus is well known to ophthalmologists. The invasion usually occurs after an injury to the corneal epithelium which may be in itself trivial, such as the trauma caused by the impaction and subsequent removal of a small foreign body. The infection usually appears. within 2 days of the injury and has a typical appearance. The lesion is a very white slough at first and later forms a dense white ring around a relatively translucent central area; if the infection has been present for more than 24 hours an hypopyon may be present.

Until recently treatment has been of little avail and either the eye has been entirely lost from perforation or all useful vision has been sacrificed because of gross corneal opacification. Recently the introduction of streptomycin and especially of the polymyxins has made the outlook less grave, as evidenced by the recent observations of Ainslie and Smith (1952) and Bignell (1951), and Williams, Hench, and Guerry (1954) have cured experimental infections in rabbits by using subconjunctival injections of Polymyxin $\mathrm{B}$ and Polymyxin E.

Because subconjunctival injection is usually a painful procedure for the patient and takes up the time of an experienced therapist, the topical application of a polymyxin potent enough to be effective when instilled into the conjunctival sac is highly desirable. An ointment has been produced containing 10,000 units Polymyxin B sulphate and 500 units Bacitractn perg. in a stable petrolatum base, and this is being tried out clinically in the Ophthalmic Hospital of St. John in Jerusalem.

A series of observations of the cornea of infected rabbits has also been carried out.

Clinical Cases.-Two typical cases have been seen of dead white ulcers with hypopyon occurring within 2 days of injury, each due to a prick by a thorn. There was no time for bacteriological confirmation of the clinical diagnosis. One ulcer healed rapidly within a week, leaving a fine white nebula and a completely quiet eye after the Polymyxin-Bacitracin ointment had been applied four times daily, that is to say after some 24 treatments in all. In the other

- Received for publication September 1, 1955. 
patient, whose age was greater and whose clinical condition was more severe, the ulcer took 10 days to heal. Those lesions are very common among the Arab population, especially during the season of the "prickly pear" harvest, when thorns commonly become lodged in the conjunctival fornix and abrade the cornea. The only additional treatment received by these patients was the twice-daily instillation of atropine ointment and the ingestion of multivitamin tablets by mouth.

Experimental Studies.-Twenty rabbits were obtained, but three were killed by snake bite and two suddenly succumbed to ether anaesthesia. In the surviving fifteen, the corneal epithelium of one eye was cut through the centre with a 2-mm. trephine and the circle of epithelium thus separated was curetted away. Some material from a known pure culture of Pseudomonas pyocyaneus was rubbed into this abraded area with a platinum loupe. Nothing further was done to the first eleven rabbits (see Figs 1-8), but Nos 12-15 were treated a few minutes after inoculation: No. 12 (Fig. 9) with an instillation of the polymyxin-bacitracin ointment, No. 13 (Fig. 10) with an application of a neomycin-cortisone ointment, No. 14 (Fig. 11) with aureomycin ointment, and No. 15 (Fig. 12) with penicillin eye ointment. The fifteen rabbits were then left alone and re-examined after 24 hours. Twelve of the fifteen were then found to have extremely severe corneal ulcers; there was a typical picture in each case of a dense white infiltration and a small hypopyon, the conjunctiva being grossly swollen and reddened with flecks of white pus on the surface. The other three rabbits (Nos 2, 12, and 13) had ulcers which were less severe. Treatment was then begun as detailed in the Table.

TABLE

TREATMENT SCHEDULE OF RABBITS $1-12$

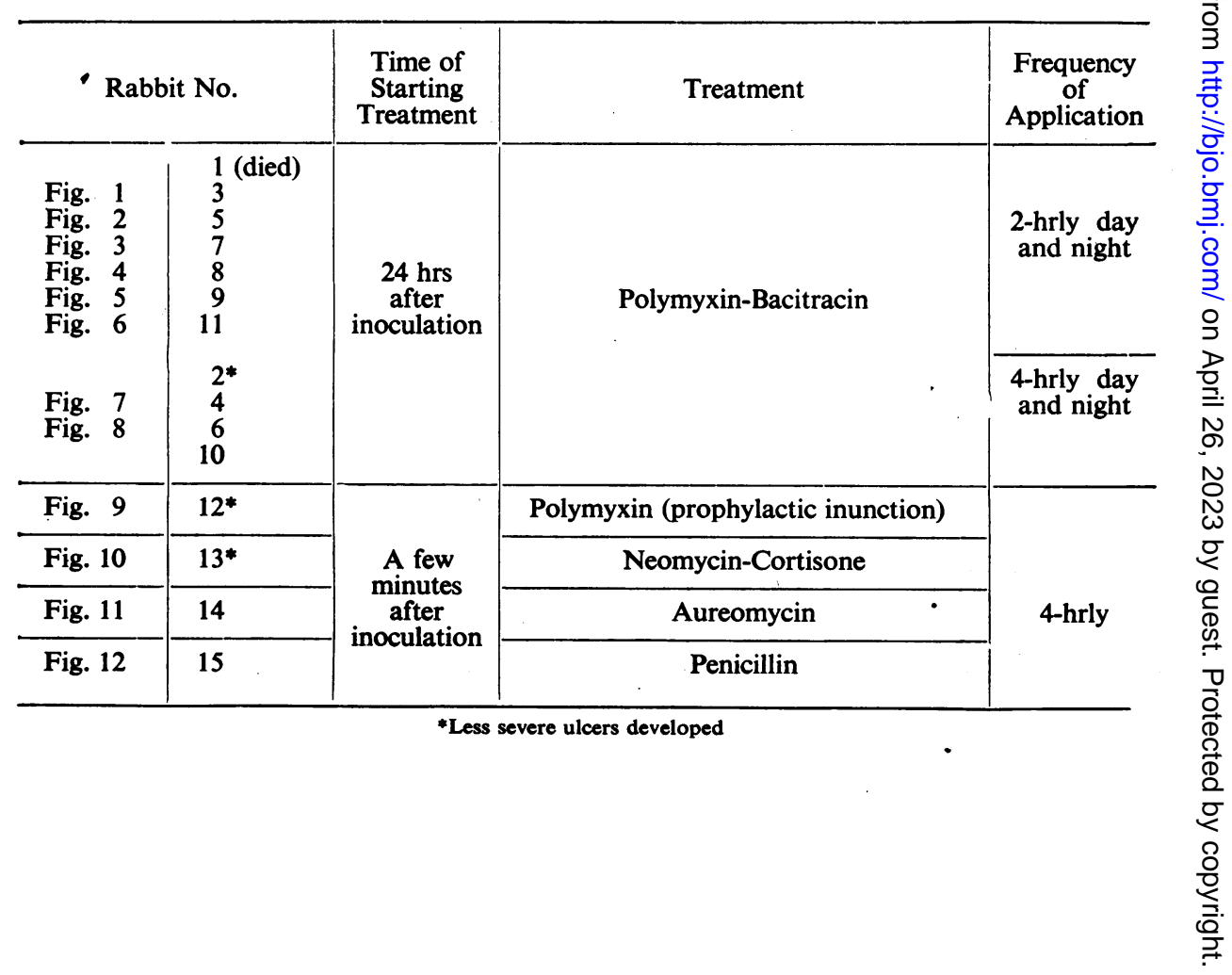




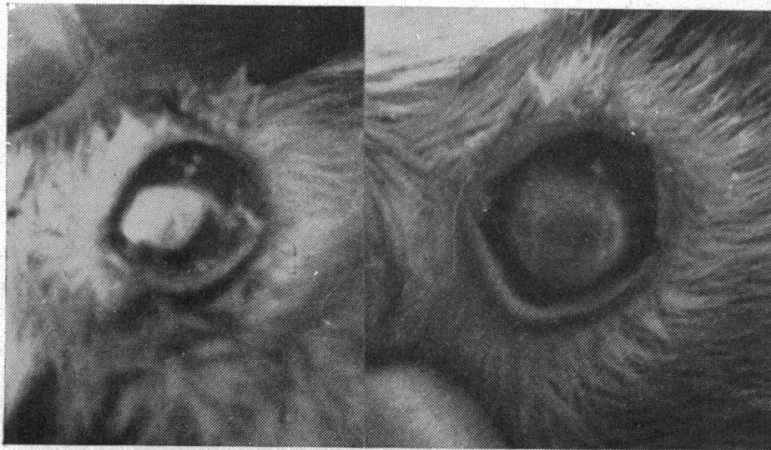

(a) (b)

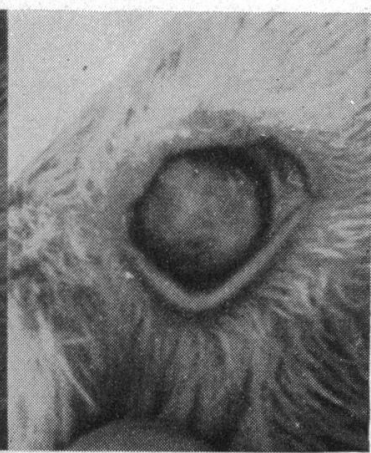

(c)

FIG. 1(a).-Rabbit No. 3, untreated, 24 hrs after infection.

FIG. 1(b).- Same eye after $48 \mathrm{hrs}$ ' treatment with Polymyxin inunctions 2 hrly, day and night.

FIG. 1(c).-Same eye after 7 days' treatment.

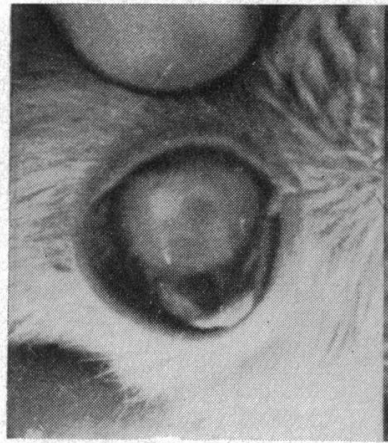

(a)

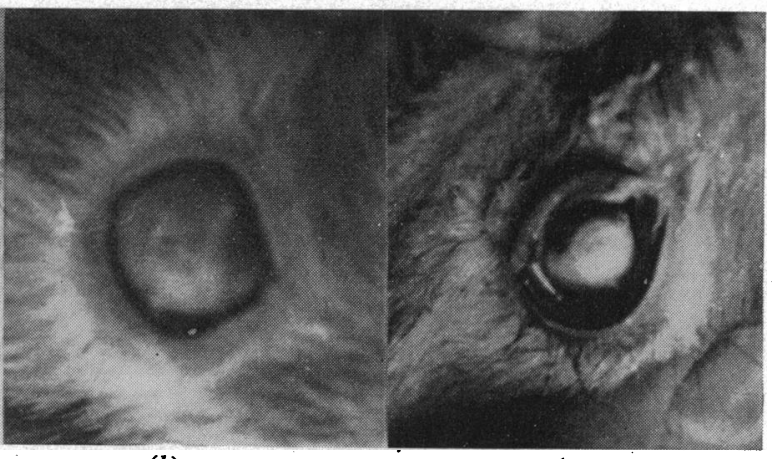

(b)

(c)

Fig. 2(a).-Rabbit No. 5, untreated, $24 \mathrm{hrs}$ after a severe infection.

FIG. 2(b).- Same eye after $48 \mathrm{hrs}$ ' treatment with Polymyxin inunctions $2 \mathrm{hrly}$, day and night.

FIG. 2(c).-Same eye after 7 days' treatment. The cornea is clearing.

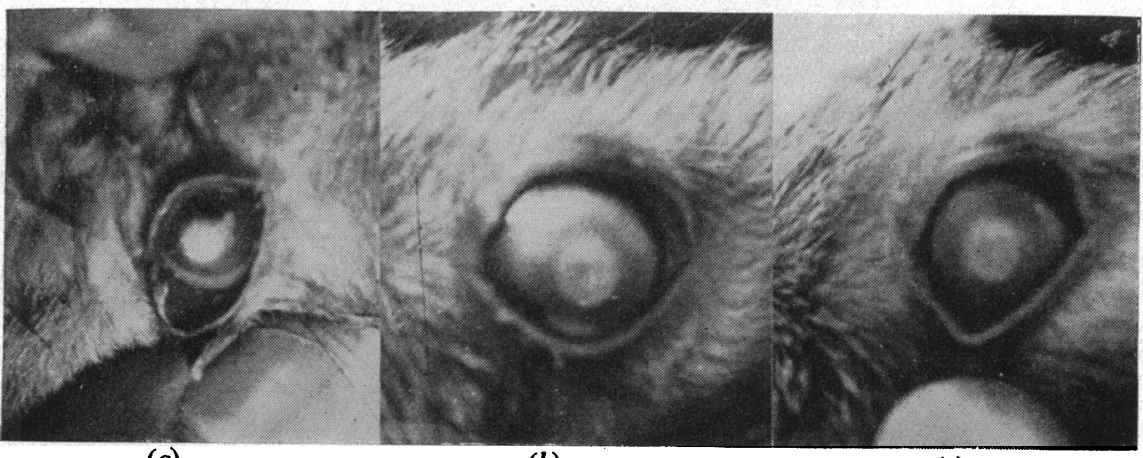

(a) (b)

FIG. 3(b).-Same eye after $48 \mathrm{hrs}$ treatment with Polymyxin inunctions 2-hrly, day and night. The ulcer is less dense.

FIG. 3(c).-Same eye after 7 days' treatment. The cornea is much clearer and the eye quiet. 


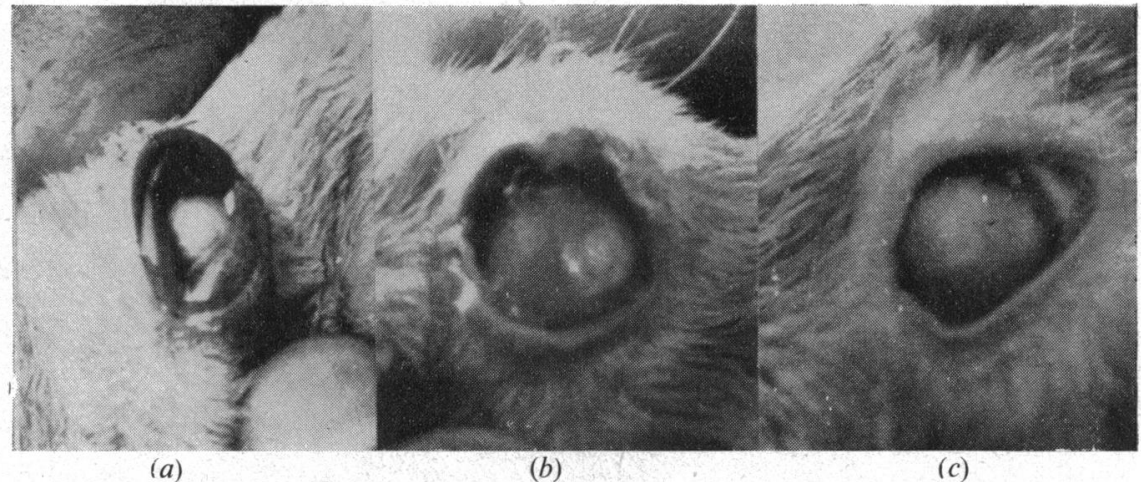

FIG. 4(a).-Rabbit No. 8, showing typical pyocyaneus ulcer, untreated, 24 hrs after infection...

FIG. 4(b).- Same eye after 48 hrs' treatment with Polymyxin inunctions 2-hrly, day and night.

FIG. $4(c)$. Same eye after 7 days' treatment. The condition is much less serious.

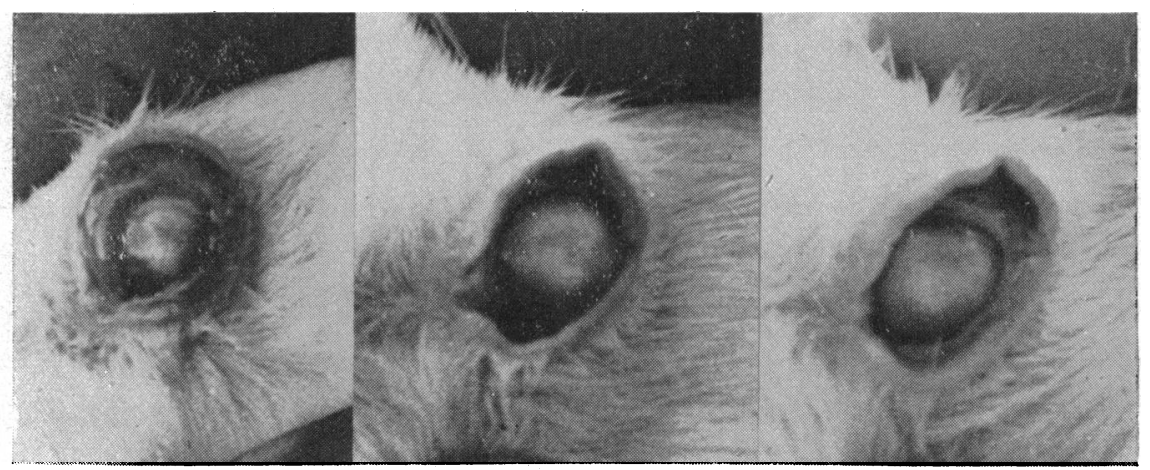

(a)

(b)

(c)

FIG. 5(a).-Rabbit No. 9, showing severe ulcer with marked infiltration and big hypopyon, untreated, 24 hrs after infection.

FIG. 5(b).-Same eye after 48 hrs' treatment with Polymyxin inunctions 2-hrly, day and night.

FIG. 5(c).-Same eye after 7 days' treatment. The ulcer is becoming much less dense and the corneal haze is disappearing at the periphery.

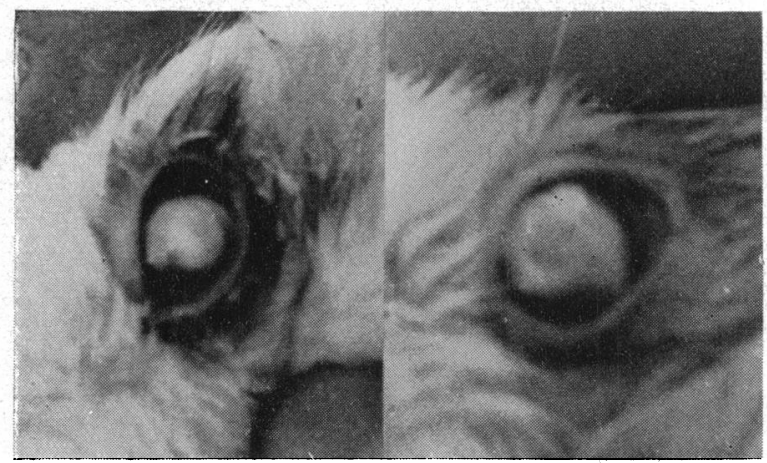

(a)

(b)

FIG. 6(a).-Rabbit No. 11, untreated, 24 hrs after infection.

FIG. $6(b)$. - Same eye after 7 days' treatment with Polymyxin inunctions 2-hrly, day and night, showing marked improvement. 


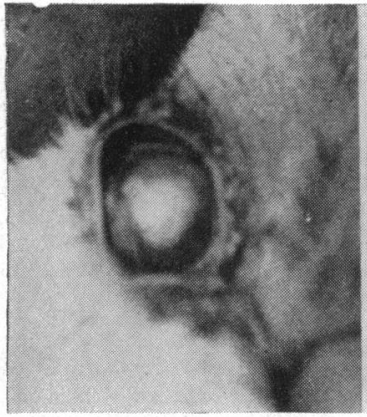

(a)

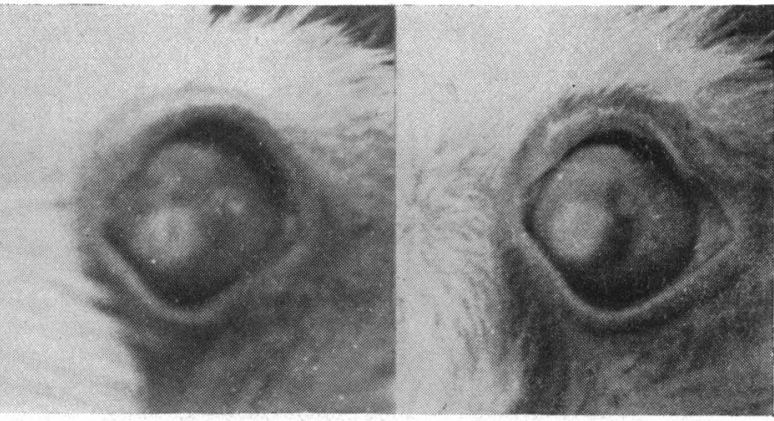

(b)

(c)

FIG. 7(a).-Rabbit No. 4, untreated, 24 hrs after infection.

FIG. 7(b).--Same eye after $48 \mathrm{hrs}$ ' treatment with Polymyxin inuctions 4-hrly, day and night.

FIG. $7(c)$.-Same eye after 7 days' treatment. The cornea is clearing.

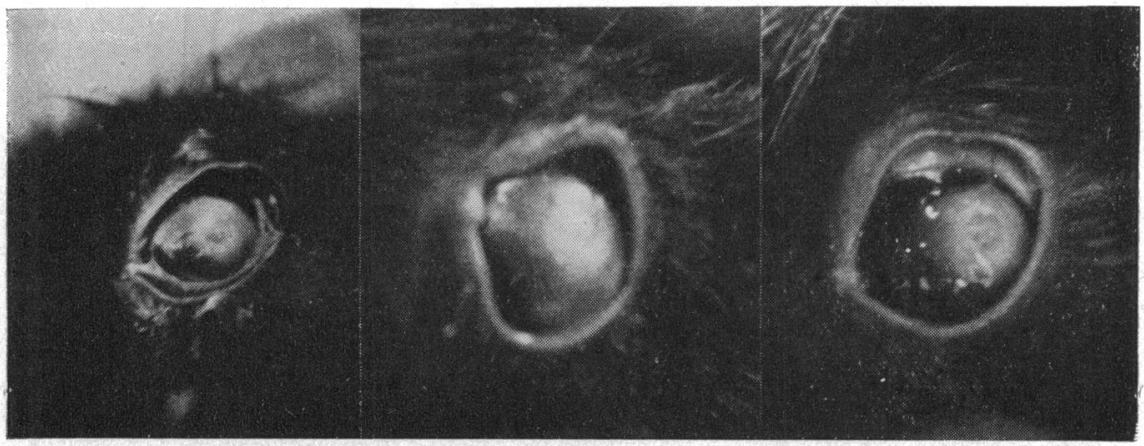

(a)

(b)

(c)

FIG. 8(a).-Rabbit No. 6, untreated, 24 hrs after infection.

FIG. 8(b).- Same eye after $48 \mathrm{hrs}$ ' treatment with Polymyxin-bacitracin inunctions

4-hrly, day and night.

FIG. 8(c).-Same eye after 7 days' treatment, showing marked clearing of the cornea.

The animals' eyes were photographed 24 hours after inoculation and before curative treatment was begun, again after having undergone treatment for $48 \mathrm{hrs,}$ and finally after having completed one week of treatment. The results after $48 \mathrm{hrs}$ were observed to be highly satisfactory in that all the eyes treated with the polymyxin ointment (Nos 1-11; see Figs 1-8) showed a marked improvement. The swelling of the conjunctiva had disappeared and the large corneal lesions were beginning to come under control. Those treated with aureomycin (No. 14) and penicillin (No. 15) remained unaffected. One treated with neomycin-cortisone ointment (No. 13), showed improvement after $48 \mathrm{hrs,}$, and this was maintained until treatment was stopped after 7 days. The vascularization of the nebulae noted by other observers was not a notable feature of these rabbits' eyes. The dense corneal infiltration became gradually more translucent and a faint bluish haze remained in areas which had previously been white and opaque. Because of the intensely hot weather the rabbits were killed on the eighth day, by which time it was considered that eight of the ulcers had healed while three showed that the infection had come under control. 


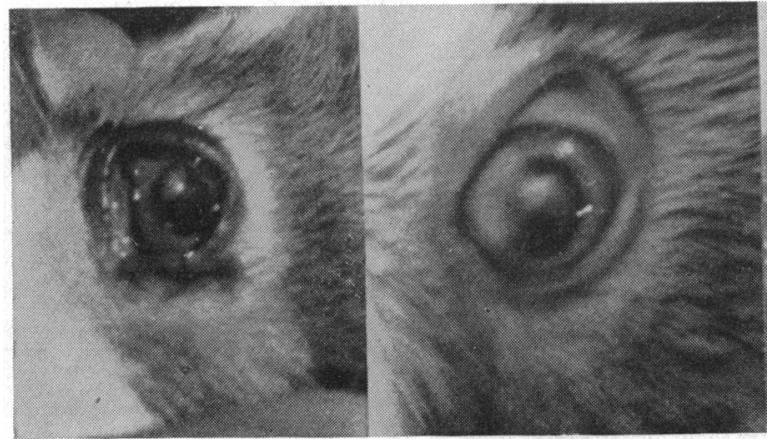

(a) (b)

FIG. 9(a).-Rabbit No. 12 (which had one prophylactic inunction of Polymyxin ointment immediately after inoculation) $24 \mathrm{hrs}$ after infection.

FIG. $9(b)$. - Same eye after 48 hrs' treatment with Polymyxin inunctions 4-hrly, day and night.

FIG. $9(c)$.- Same eye after 7 days' treatment. Improvement is obvious.

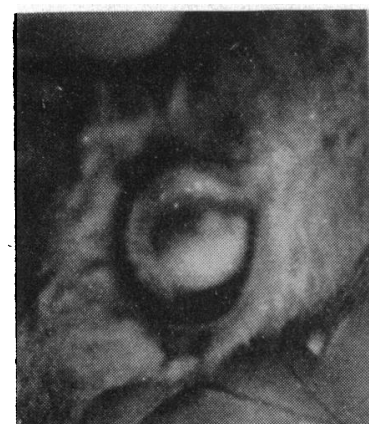

(a)

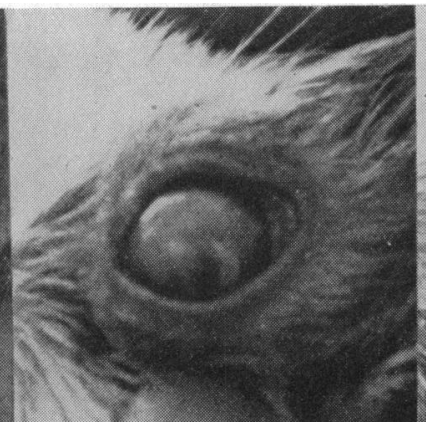

(b)

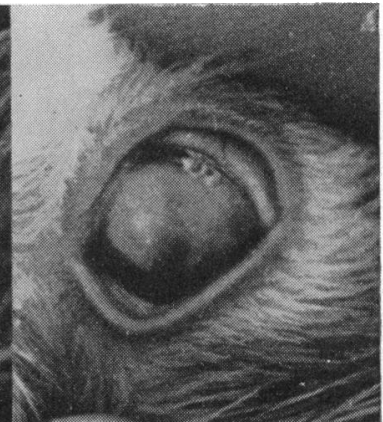

(c)

FIG. 10(a).-Rabbit No. 13 (which had one prophylactic inunction of neomycincortisone ointment ("Neosone") immediately after inoculation) $24 \mathrm{hrs}$ after infection.

FIG. 10(b).-Same eye, after 48 hrs' treatment with " Neosone" 4-hrly, day and night, is much improved.

FIG. 10(c).-Same eye after 7 days' treatment. The cornea is clearing well.

\section{Discussion}

It was clear from these observations that the frequent topical application of an ointment containing Polymyxin B and Bacitracin has a beneficial effect upon severe corneal lesions induced in rabbits by the inoculation of Pseudomonas pyocyaneus into corneal abrasions. A prophylactic inunction with the ointment after the infection of one cornea (No. 12, Fig. 9) appeared to reduce the severity of the lesion but did not completely prevent it. The corneae treated by inunctions of aureomycin ointment and penicillin ointment responded little if at all. It would also appear from these observations that the application of Polymyxin B locally to the eye in ointment form would be useful in the treatment of pyocyaneus infections in human subjects. Two typical but unconfirmed lesions in human patients responded rapidly to these inunctions. As the pyocyaneus infection frequently follows corneal trauma, 


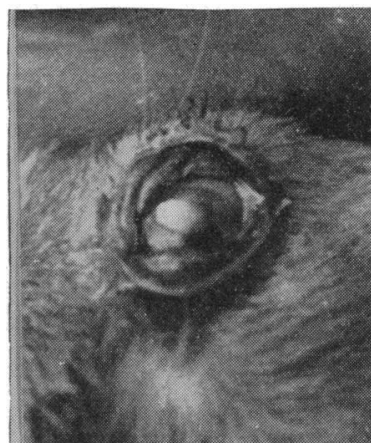

(a)

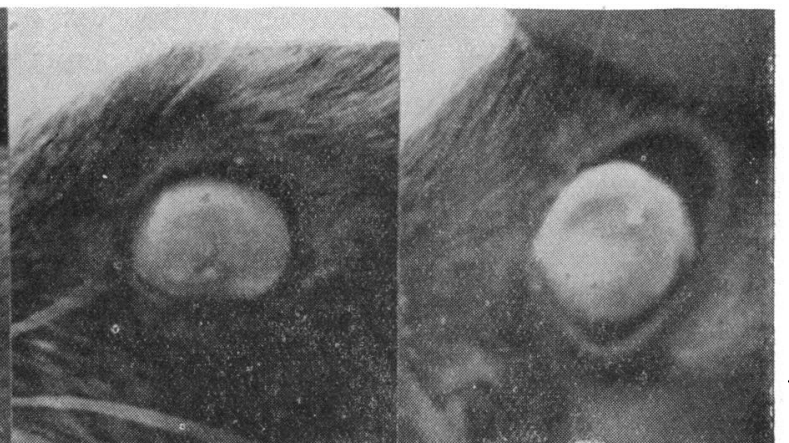

(b)

(c)

Fig. 11(a).-Rabbit No. 14 (which had one prophylactic inunction of aureomycin ointment immediately after inoculation) $24 \mathrm{hrs}$ after infection.

Fig. 11(b). - Same eye after 48 hrs' treatment with aureomycin inunctions 4-hrly, day and night. The cornea is extensively affected.

Fig. 11(c).-Same eye after 7 days' treatment. The eye is in a very bad state.

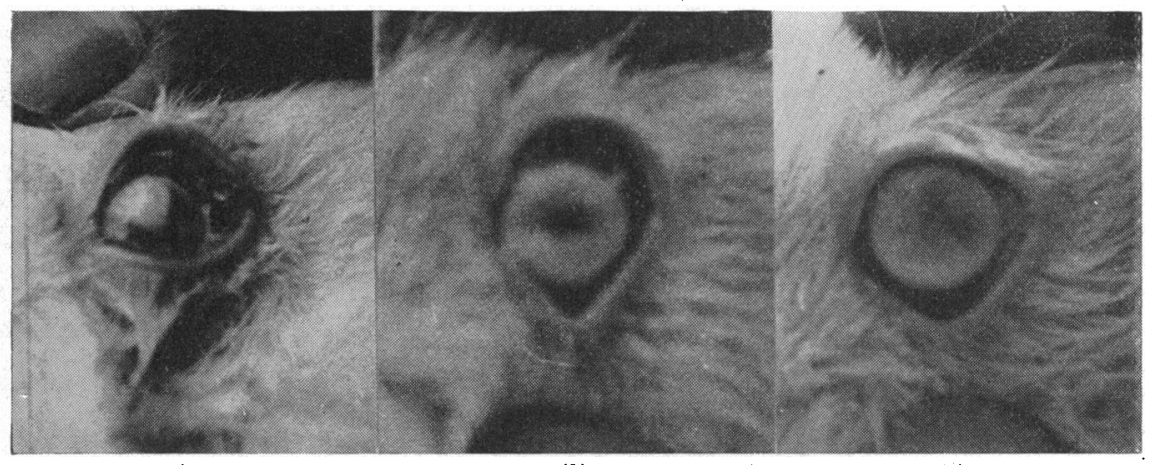

(a)

(b)

(c)

FIG. 12(a).-Rabbit No. 15 (which had one prophylactic inunction of penicillin ointment immediately after inoculation) $24 \mathrm{hrs}$ after infection.

FIG. 12(b).--Same eye after $48 \mathrm{hrs}$ ' treatment with penicillin inunctions 4-hrly, day and night.

FIG. 12(c).-Same eye after 7 days' treatment.

the local application of polymyxin ointment would be useful as a prophylactic measure in out-patient ophthalmic clinics and in industrial first-aid departments. Treatment should not be deferred pending identification of the organism. The role of the laboratory here is confirmatory and not diagnostic.

\section{Summary}

(1) Two clinically typical but bacteriologically unproven cases of pyocyaneus infection of the cornea responded promptly and satisfactorily to local applications of an ointment containing 10,000 units Polymyxin B and 500 units Bacitracin per $\mathrm{g}$.

(2) Eleven rabbit corneae inoculated with Pseudomonas pyocyaneus re- 
sponded well to 7 days' treatment with this ointment. The Polymyxin ointment was superior to aureomycin and penicillin.

(3) Frequent applications of the ointment are likely to control pyocyaneus infections of the cornea in man, and ointment should be used as a prophylactic in cases of corneal injury, especially those caused by foreign bodies in industry.

My thanks are due to Surgeon Vice-Admiral Sir Alexander Ingleby-Mackenzie, K.B.E., C.B., Q.H.P., Medical Director-General of the Navy, and to Sir Stewart Duke-Elder, K.C.V.O., Hospitaller of the Order of St. John, for permission to publish this paper.

The Polymyxin-Bacitracin ointment, kindly supplied by the Wellcome Research Foundation, is produced under the trade name of "Polyfax".

\section{REFERENCES}

AINSLIE, D., and SMITH, C. (1952). British Journal of Ophthalmology, 36, 352.

BIGNELL, J. L. (1951). Ibid., 35, 419.

Williams, R. K., Hench, M. E., and Guerry, Du Pont (1954). Amer. J. Ophthal., 37, 538. 